\section{Using Minecraft as a Geodesign Tool for Encouraging Public Participation in Urban Planning}

Gl_Forum 2017, Issue 1

Page: 200 - 314

Short Paper

Corresponding Author

AhmedHanie.Elmerghany@edu.fh-

kaernten.ac.at

DOI: 10.1553/giscience2017_01_s300

\author{
Ahmed Hanie Elmerghany and Gernot Paulus \\ School of Engineering \& IT, Department of Geoinformation and Environmental \\ Technologies, Carinthia University of Applied Sciences, Villach, Austria
}

\begin{abstract}
Simulation of reality in computer games has recently been adopted for urban planning purposes. Minecraft ${ }^{\mathrm{TM}}$ is a computer game in which players use $1 \mathrm{~m}^{3}$ blocks of various materials to construct buildings in an imaginary world. It has become popular recently as a geodesign tool for encouraging public participation in urban planning. As part of this research, a prototype model was developed based on the enhanced transformation of actual spatial datasets into a Minecraft virtual world. A Minecraft-based, collaborative geodesign participatory process was conducted in order to assess a geodesign proposal for a location within the study area in Austria. The research aims to explore the usability of Minecraft as a geodesign tool that can facilitate public participation in urban planning decision support. The results showed that maintaining adequate realism in Minecraft model designs can positively influence users' sense of orientation and improve their navigation in a Minecraft world. A disadvantage of using Minecraft is that assessing the reliability of designs for urban planning is time-consuming. Furthermore, using Minecraft models requires participants to be highly motivated
\end{abstract}

\title{
Keywords:
}

geodesign, minecraft, public participation, urban planning, 3D GIS, LiDAR

\section{Introduction}

Geodesign is an iterative design method that uses stakeholders' input, geospatial modelling, impact simulations, and real-time feedback to facilitate holistic designs and smart decisions (McElvaney \& Foster, 2014). Input from various stakeholders, like urban planners, architects, decision makers and public participants, is a key element. There is a significant need to provide simple and innovative design tools that can satisfy the expectations of diverse potential stakeholders who have varying expertise.

Geodesign incorporates active and proactive approaches of changing models (Breuer et al., 2014). This research adopts a proactive changing model environment, aiming to improve the existing conditions within a real geographic location. The major focus of this research is to 
assess input from public participation (PP), based on the so-called gaming-in-geodesign concept. Previous experience has shown that gaming can be effective in supporting decision making for sustainable urban planning (Mayer et al., 2005). Gaming in geodesign allows multidisciplinary stakeholders to provide a clear vision of their needs in a collaborative way (ibid.).

One of the most popular games that have been used recently in geodesign is Minecraft. According to Coudray (2015), Minecraft had over 100 million players around the world in 2014. Minecraft encourages the creativity of the players to construct objects from their own perspectives (Duncan, 2011).

\section{Related Work}

Minecraft has been used in several participatory processes as a platform for collaboration among citizens, so that they can provide suggestions for an urban design process (Amado et al., 2010). In a recent case study in Norway, Helleborg \& Åmdal (2015) developed a system called Real Terrain that facilitates generating Minecraft worlds using open geospatial data. The purpose of their system was to test the users' interpretation and recognition of the created Minecraft world. According to Helleborg \& Åmdal (2015), most of the participants used a water feature as a reference when trying to identify the particular location. Results of their research also indicated that children and teenagers had difficulties in navigating the Minecraft world, unlike the group of early college students.

Another case study was based on re-designing a public space in the city of Kirtipur in Nepal (von Heland et al., 2015). The study incorporated a Minecraft workshop to involve habitants of Kirtipur in an interactive participatory design process for a public park. Statistical results of the qualitative interviewing with various groups of participants showed that Minecraft increased interactivity in urban design processes among young participants.

'Denmark in Minecraft' is a project run by the Danish Geodata Agency and is sponsored by the government of Denmark. The project aimed to create a 3D virtual world of Denmark in Minecraft, based on transforming a Digital Terrain Model (DTM) as well as cadastral data of Denmark into a Minecraft world (Denmark in Minecraft, 2016). Additionally, the transformation of a 3D city model using Geography Markup Language (GML) and 3D architectural models resulted in realistic structures not only at the city scale but also the at the scale of individual buildings in the Denmark Minecraft model. The goal of the project was to engage young students in urban planning, and to utilize Minecraft in education (Magnussen \& Elming, 2015).

\section{Goal and Research Problems}

The goal of this research is to explore the applicability of Minecraft as a geodesign tool for facilitating collaborative public participation in urban planning. The main focus is to enhance the realism of Minecraft models by the automated integration of high quality spatial datasets such as Light Detection and Ranging (LiDAR) point clouds, as well as classical Geographic Information Systems (GIS) datasets. The challenge in the proactive geodesign approach adopted in this research is the formulation of design proposals that start from an existing 
condition in order to develop a future plan. The process of engaging public participants in a collaborative way to assess designs proposed by inhabitants for a selected area adds to the complexity of geodesign.

There are still limitations and weaknesses to overcome in order to improve outcomes of public participation using Minecraft and urban planning. Vague representation of geospatial data leads to users facing difficulties navigating in the Minecraft world (Rautenbach et al., 2014). In order to improve the users' orientation, visualization of the geospatial data simulating a real-world location in Minecraft should include distinct topographical features, landmarks and points of interest (Formosa, 2014). Research questions addressed here, therefore, are: 1) Can Minecraft provide a reliable geodesign proposal for urban planning purposes by encouraging collaborative public participation?; 2) Can typical high resolution spatial data like LiDAR and land cover/land-use data be automatically transformed into virtual Minecraft worlds in order to provide better and more realistic base representations for geodesign?

\section{Methodology}

\section{Overview}

The creation of the Minecraft prototype involved integrating pre-processed datasets covering the selected study area in the city of Villach, Austria. In order to produce a detailed Minecraft terrain, high resolution DEM is a prerequisite as a basic input raster feature. The DEM is retrieved from a classified LiDAR point cloud during the pre-processing workflow (Formosa, 2014).

Terrain objects in a Minecraft world have a unique block-ID and location. Therefore, the data transformation workflow enables the conversion of the raster pixel values from the DEM into Minecraft block-ID. The data transformation workflow also includes adding a block-ID as an attribute to the given datasets, and the conversion of vector layers such as land use and building footprints into Minecraft point-cloud format. Transforming geospatial data into a Minecraft world involves assigning realistic block-ID values for the landscape features so that they simulate the real-world location - for instance, assigning the grass block-ID '2' to vegetation features (Dmitribagh, 2015).

After the datasets have been converted to Minecraft point cloud format, constructing the terrain regions takes place through the Minecraft game engine, and thereafter a Minecraft output world is generated. To finalize the prototype design process, the generated Minecraft world is validated through the user-participation process. The purpose of the participation process is to obtain design proposals from the volunteer participants that will be retained. These geodesign proposals are then re-transformed into point cloud format for featuressplitting by new generated block-ID values in order to map the design proposals. A further result from this stage is participants' feedback concerning the usability of Minecraft as a geodesign tool. Figure 1 shows an overview of the data integration workflow for the creation of a Minecraft prototype, in which volunteer participants' design proposals are then assessed. 


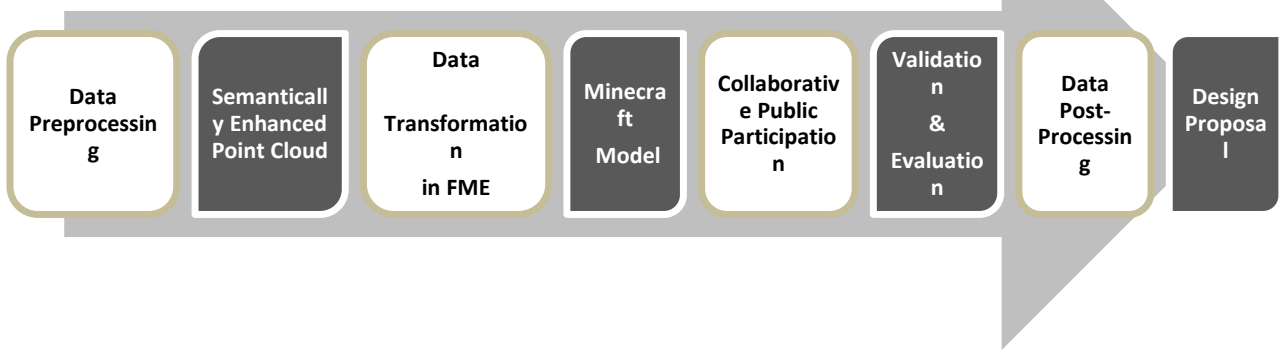

Figure 1: Overview of workflow for data integration and geodesign assessment

\section{Study Area}

The study area is located in the city of Villach in Austria, where the campus of Carinthia University for Applied Science is located (Figure 2). This area comprises various landscape features to be transformed into a Minecraft prototype world, such as rivers, vegetation, builtup areas and transport networks.

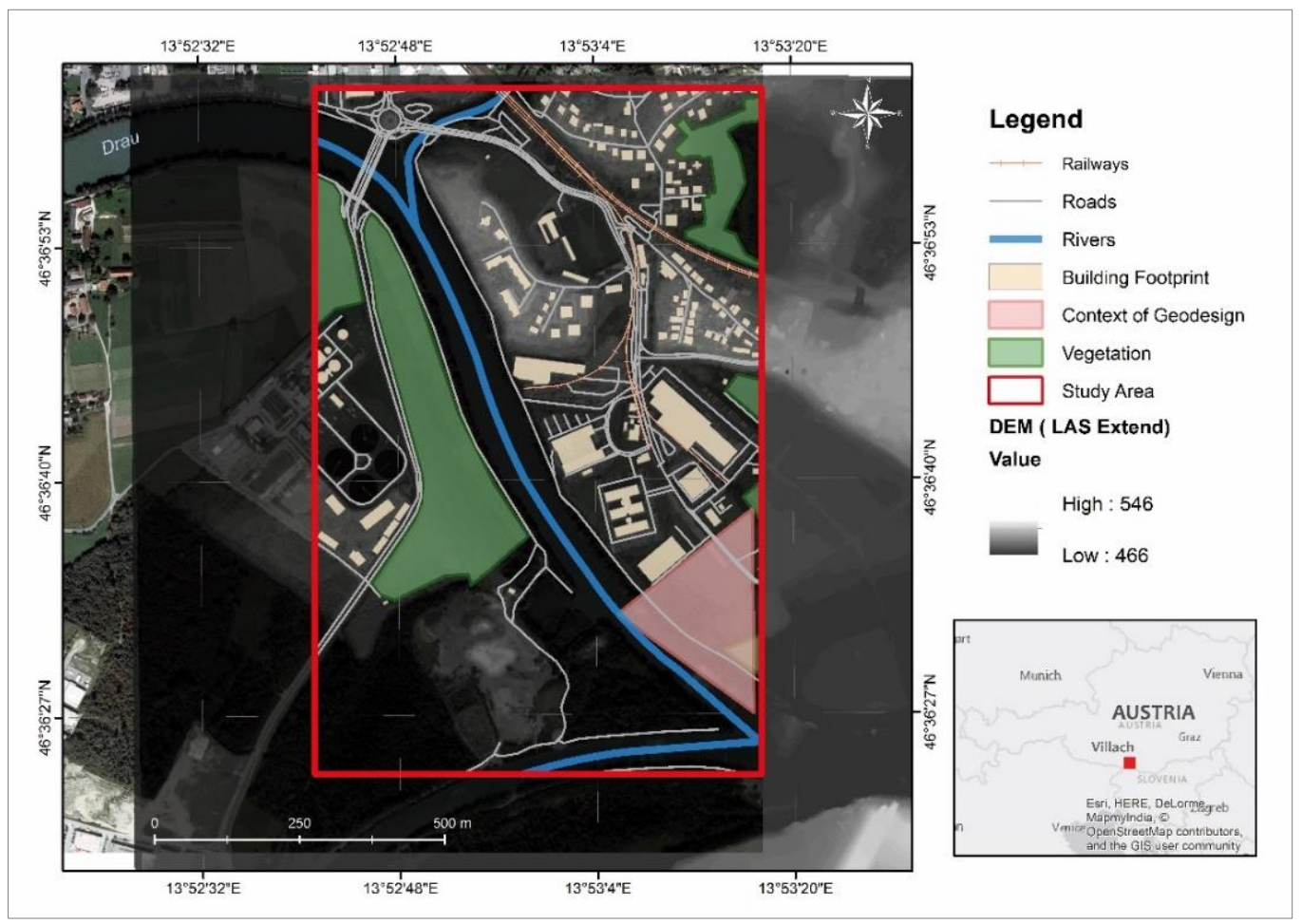

Figure 2: Integrated datasets within the study area 


\section{Datasets}

A Minecraft world is a point cloud that can be generated from geospatial data; Minecraft block-ID and block-Data attribute values are assigned to each point. Each block-ID reflects a natural colour from the real world. Creating a Minecraft world is an automated process of integrating several datasets (Figure 2).

The LiDAR point cloud, provided by the Carinthian Geographic Information System KAGIS, covers the eastern part of the city of Villach in Austria, and consists of 17,557,078 laser scan points. The LiDAR point cloud is the primary input for producing the output Minecraft world. Essential features such as a DEM, vegetation and 3D buildings are extracted from the LiDAR point cloud.

Open Street Map (OSM) layers obtained from Geofabrik (Geofabrik, 2015), including road networks, rivers, railways, lakes, land use and building footprints, are used to supplement the LiDAR point cloud in order to improve the aesthetic appearance of the Minecraft prototype. Moreover, a high resolution orthophoto was used as a colourization reference to enhance the primary LiDAR point cloud.

\section{Minecraft Prototype Design}

Input datasets are integrated and combined using FME ${ }^{\mathrm{TM}}$ in order to produce the Minecraft prototype. The potential of FME, an integrated collection of spatial Extract, Transform and Load (ETL) tools for data transformation, is its ability to write and read Minecraft point cloud format.

\section{Pre-processing}

Before data transformation and integration, each dataset is run independently through a series of geoprocessing operations (Figure 3).

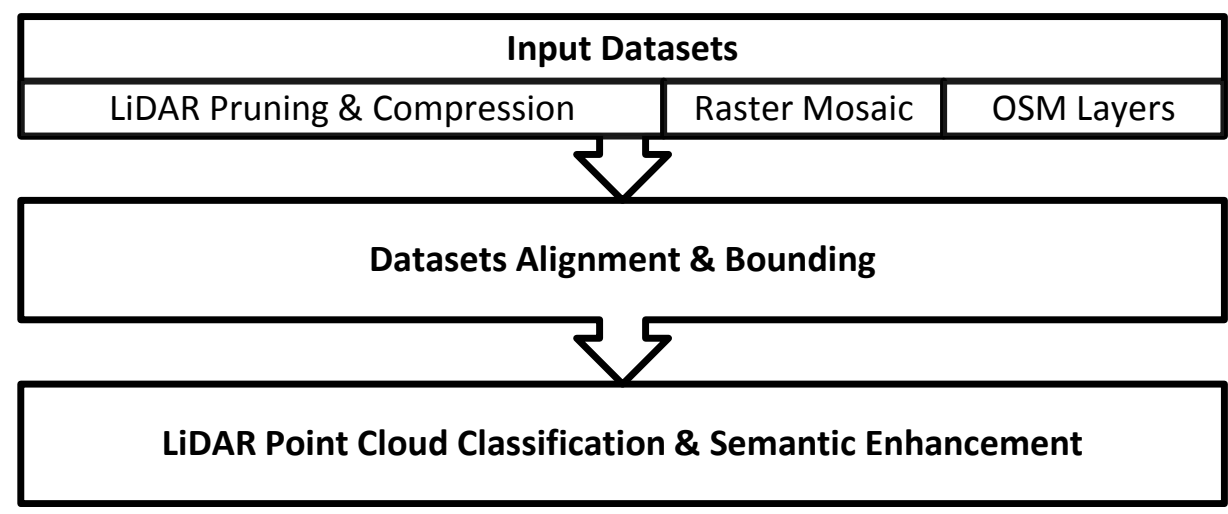

Figure 3: Data pre-processing workflow for LiDAR point cloud enhancement 
Due to extreme outlier points, the extent of the LiDAR point cloud was expanded and skewed. In order to limit this expansion, the original point cloud was pruned and outlier points were removed. The pruned LiDAR point cloud is then compressed to LAZ format using LAS writer in FME. LAZ compression allows minimizing the LiDAR point cloud to $13 \%$ of its original file size without the loss of any points, and helps to reduce the data translation time.

Mismatching between input features leads to the failure of data transformation and results in the absence of terrain objects in the anticipated Minecraft prototype. Therefore, matching and downscaling OSM layers as well as the orthophoto to the extent of the study area, and georeferencing them to the exact spatial reference and geographic location of the LiDAR point cloud are perquisites for the implementation phases that follow. Figure 4 shows the alignment of input datasets after georeferencing.

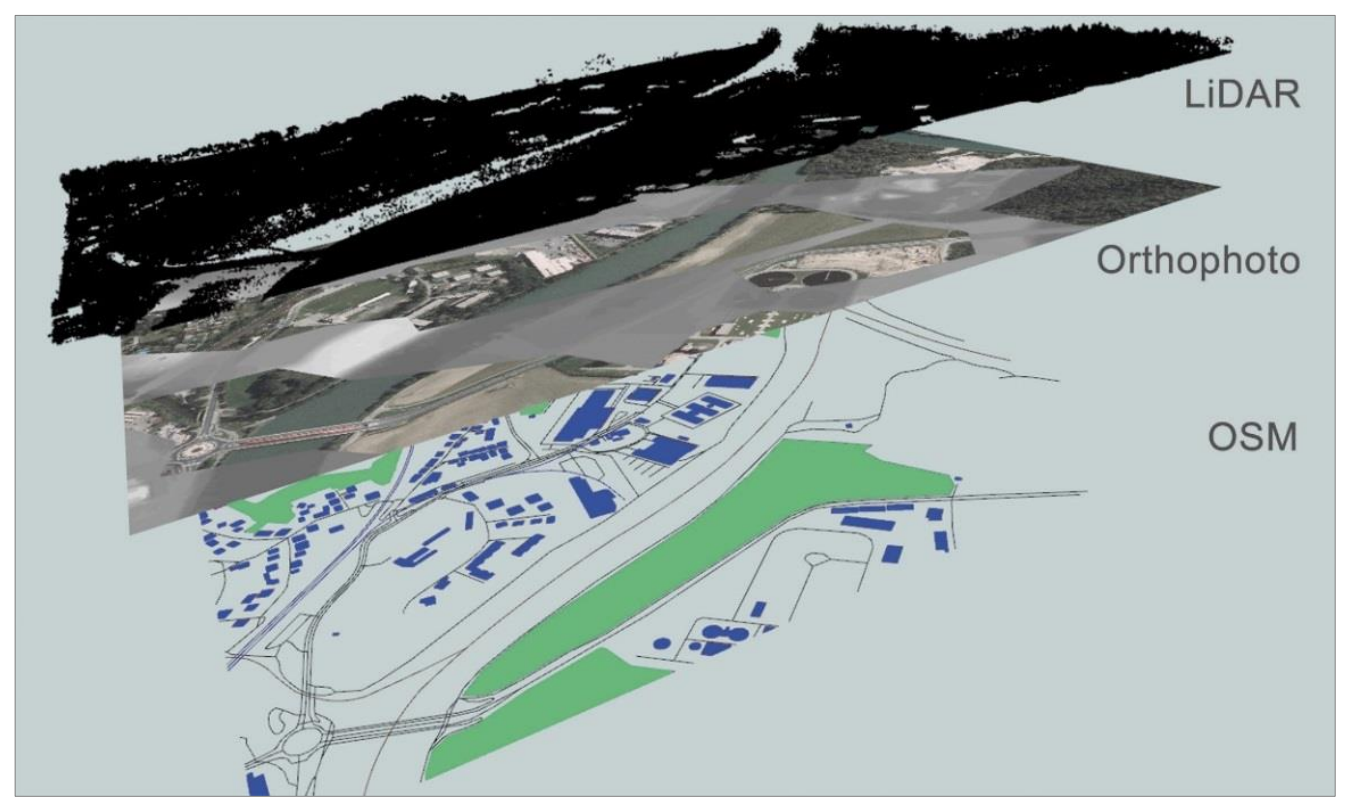

Figure 4: Alignment and bounding of input datasets

Classified point cloud features are gathered in a single point cloud, which includes precise building-class points, high and medium vegetation-class points, and ground-class points. The partial LiDAR classes enable the extraction of DEM from class-2 points, 3D buildings from class- 6 points, and vegetation from class-5 points.

LiDAR point cloud classification using LAS Tools ${ }^{\text {TM }}$ custom transformers in FME resulted in the removal of noise points. Points are classified as class-2 ground, high vegetation points as class 5, and building points as class 6 , according to ASPRS standardized point cloud classification. 
Classification of buildings was sometimes inaccurate due to misclassification of some building points. In order to obtain the accurate classification of buildings, building points in class 6 were filtered within the overlaid OSM building footprints.

\section{Data Integration and Transformation}

Partial point clouds are generated in FME by specifying the split-value parameter based on LiDAR point classification values. Multiple intermediate processes are applied upon each point cloud separately. The generated DEM from the ground class manipulation replaces the original coordinate system with number of rows and columns (Figure 5). The buildings point cloud goes through a series of extrusion and filtering operations. Likewise, each input feature from OSM vector layers goes through categorization, sorting and finally buffering in order to produce explicit point clouds that can be styled individually to simulate each anticipated terrain object in the final Minecraft world.

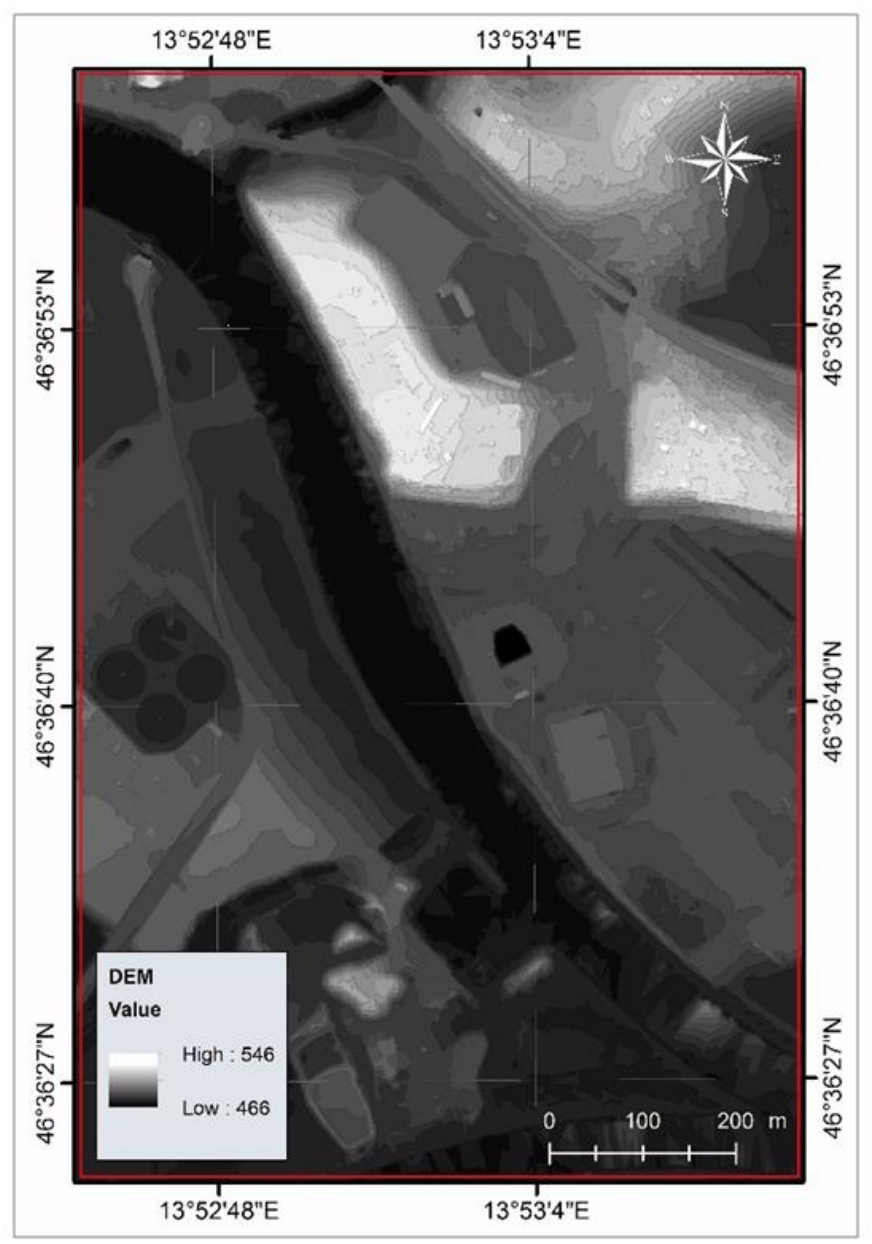

Figure 5: DEM generated from LiDAR point cloud 
Creating a Minecraft world with the actual coordinates of the DEM results in a massive Minecraft world that fails to generate a useable Minecraft region. The Minecraft game engine limits the "Z" range between 0 and 255. Therefore, replacing the real coordinates with numbers of rows and columns reduces the Minecraft terrain vertical volume.

Thereafter, the DEM raster is transformed into a ground point cloud as a base layer for the final Minecraft terrain.

Extracting 3-D buildings geometry involves integrating the point cloud for buildings (class 6) with the OSM building footprints. The lower and upper bounds of each 3-D building geometry are determined by the point cloud statistics for the minimum and maximum " $\mathrm{Z}$ " values respectively within each building footprint.

The extracted output of buildings is then turned then in the form of an individual point cloud for each building within the study area. These point clouds can be filtered and colourized individually to improve the appearance of the final Minecraft prototype.

Intermediate processing is performed simultaneously upon additional OSM layers to compensate for missing features from the original LiDAR point cloud. Additional features included transport networks, rivers and lakes. Each feature is manipulated separately, in order to be combined with all other input features in the definitive point cloud, from which the final Minecraft world will be generated. For instance, transport network features are categorized into three different types of roads, according to the OSM "type" attribute values. The road types are major roads, cycle ways and secondary roads, which include service and residential ways.

The elevation of each individual point cloud is adjusted to the actual terrain height according to the generated DEM from the original classified point cloud for output refining. Subsequently, the elevations of terrain objects are sorted from lowest to highest. Moreover, the colourization process specifies the appearance of each input feature in the final Minecraft prototype. Determining the appearance of terrain objects in Minecraft was handled by specifying block-ID and block-Data for each partial point cloud. Thereafter, all individual point clouds are combined into a single comprehensive point cloud that represents the output Minecraft point cloud. The final step is building a terrain from multiple regions that will form the final Minecraft prototype of the study area. Figure 6 shows the reference standard Minecraft blocks (Minecraftinformation.com, 2012) used for 3D visualization of multiple terrain objects in Minecraft, simulating the actual location of the study area in the real world. 


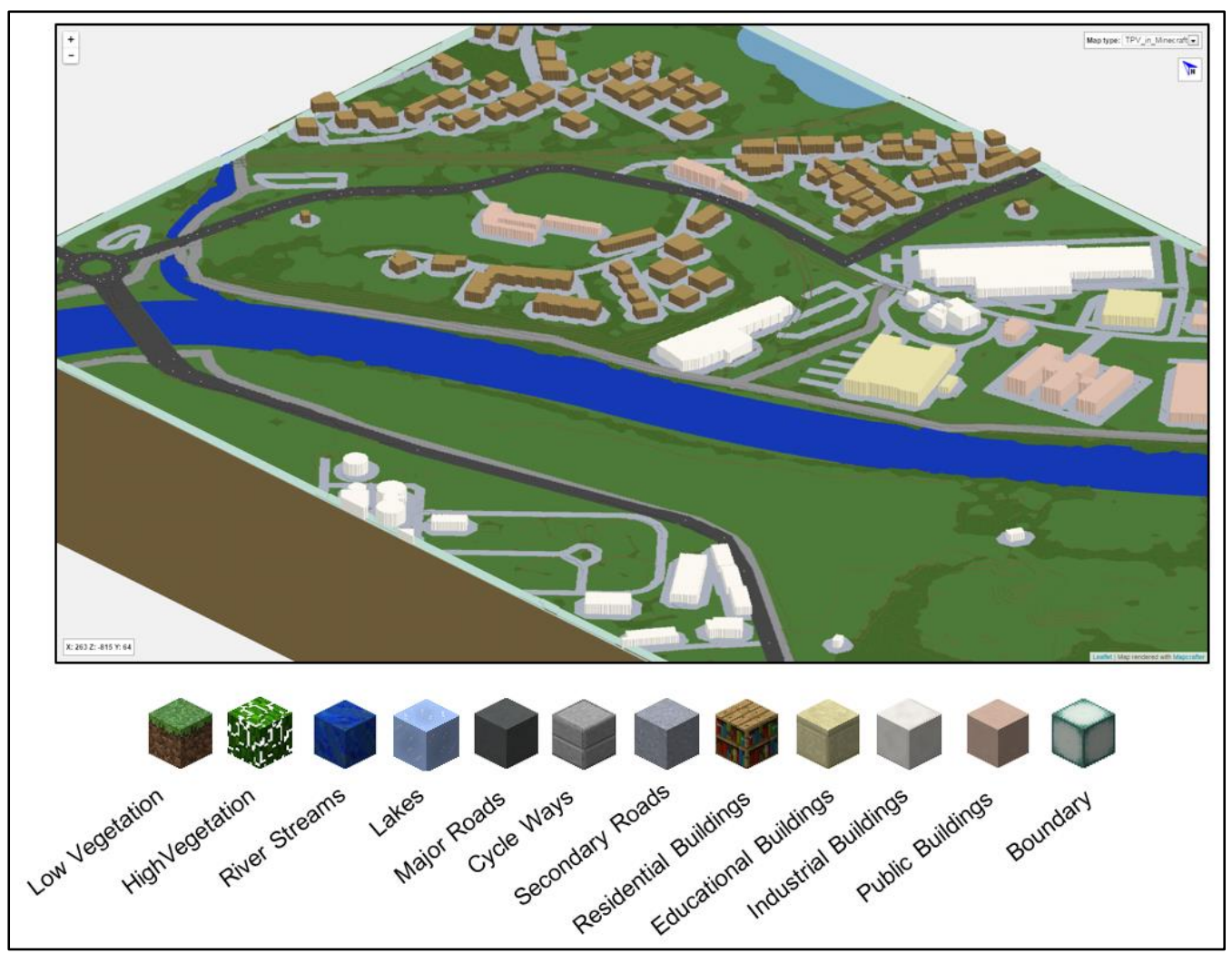

Figure 6: Minecraft world of the study area in Villach, Austria.

\section{Collaborative Geodesign Participation Process}

The purpose of the participation process is to acquire design proposals from volunteer participants. A significant outcome from the participatory process is the users' feedback about the usability of Minecraft as a geodesign tool for urban planning.

A one-day Minecraft geodesign participatory workshop took place at Carinthia University for Applied Sciences in Villach, Austria. Four students aged 24-28, from various educational backgrounds, attended the workshop. The participants were invited to elaborate their design ideas for developing a preselected site on the university campus.

The targeted area as a context of geodesign was an undeveloped plot of land used only as parking space, while it could be used for the expansion of the university campus.

Prior to working on the design process itself, participants were guided through a training session covering the game controls and the interaction between players within a shared Minecraft prototype world. The training session also included selected video tutorials as well a reference guide for Minecraft blocks (Minecraftinformation.com, 2012) that could be used during the design process. Thereafter, participants were invited to explore the shared 
Minecraft prototype world to ensure that they understood the Minecraft game instructions and use of the game controls before starting the design proposal implementation. Following the training session, participants started to plan a design proposal for expanding the university. A brainstorming session resulted in a list of suggested facilities to be constructed in the Minecraft prototype during the design process. Participants sketched a paper draft of the design proposal using an orthophoto image as a reference for the context of geodesign.

Thereafter, the design process involved collaboration among all participants in some common tasks, such as extending roads and placing street furniture, although it was noticeable that each participant took individual responsibility for the construction of a particular building independently. Participants also exchanged roles and tasks while building the pre-identified suggestions.

The major challenges during the design process were the lack of daylight, due Minecraft's short day length, as well as the changing weather conditions, from clear to rainy, in the Minecraft world. Moreover, the lack of a sense-of-orientation resulted in players not being able to instantly recognize their locations in relation to other players in the virtual Minecraft world. In order to mitigate such problems during the design process, participants were instructed to use specific game command lines for extending the Minecraft daylight time, instant teleporting and changing weather conditions, which helped in improving design efficiency.

\section{Participation Feedback and Evaluation}

After completion of the collaborative design sessions, an online survey requested participants' feedback and evaluation of the use of Minecraft as an urban planning design tool. The first part of the survey (Appendix A) reflected participants' evaluation of the Minecraft prototype from multiple aspects, by answering targeted questions such as: 1) Are 3D box-like buildings helpful for a better sense of orientation?; 2) How would you describe the navigation in Minecraft?; 3) How would you describe the sense of orientation in Minecraft?; 4) Does the Minecraft model look like the reference map?

Analysing the results from the first survey showed that on a scale of 1 (easy) to 5 (difficult), $50 \%$ of the participants described navigation and moving between locations during the design process as easy, and 50\% found it relatively difficult. Likewise, participants rated sense of orientation on a scale of 1 (simple) to 5 (complicated). $50 \%$ of the participants rated sense of orientation and recognition of current location as above average (fairly simple), while $25 \%$ of the participants found it average, and $25 \%$ found it below average (fairly complicated).

In order to assess the realism level in Minecraft prototype, the participants were shown an aerial view of the Minecraft prototype as well as a satellite image of the same view from Google Earth ${ }^{\mathrm{TM}}$ for visual interpretation. 50\% of the participants agreed that the Minecraft prototype looked similar to the real terrain in the given reference map, and $50 \%$ mostly agreed.

The participants were asked about the impact of the 3D buildings in Minecraft on their sense of orientation. $50 \%$ of the participants agreed that they were helpful for a better sense of 
orientation, while $25 \%$ found their presence above average (agreed to an extent) and $25 \%$ were neutral (neither agreed nor disagreed).

To evaluate the efficiency of using Minecraft in geodesign, the participants were asked multiple questions, such as: 1) How familiar are you with Minecraft?; 2) Teamwork when building in Minecraft went well; 3) I can represent my design ideas in Minecraft; 4) Minecraft is a suitable tool for geodesign; 5) I would participate in a similar Minecraft Geodesign workshop again.

The results of the Minecraft efficiency evaluation (Appendix B) showed that although most of the participants did not have previous experience of Minecraft, the majority of them believed that Minecraft could be a good tool for geodesign. $75 \%$ of participants were in general agreement, and $25 \%$ completely agreed that Minecraft is a suitable tool for geodesign. Additionally, the participants were asked to rate their opinions concerning the conflicts that could arise due to the interactions of multiple players while building in Minecraft. All participants agreed that teamwork during the collaborative design sessions was helpful and fruitful. All participants agreed that they received sufficient training during the workshop to enable them to build their design ideas in Minecraft.

The Minecraft usability evaluation addressed the participants' motivation to take part in similar Minecraft workshops for geodesign and urban planning purposes. $50 \%$ of the participants stated that they would participate again in similar events, while $25 \%$ felt that they probably would participate, and 25\% didn't know and were neutral. The open discussion following the online survey allowed further assessment of the participants' insights and their overall impressions about the workshop and using Minecraft for geodesign. The participants were satisfied with the framework of the workshop. They found that the collaborative design in Minecraft was successful and fruitful, and cooperation during the design process in Minecraft was not complicated.

However, the small number of participants in this study can provide only general indications about the prototype design and the overall workflow (Figure 1). We recommend that the collaborative participation workflow introduced in this research be implemented in multiple iterations, with a larger number of participants, in order to derive valid conclusions.

\section{$5 \quad$ Results}

The post-processing workflow in Figure 7 illustrates the handling of the Minecraft point cloud after completion of the public participation process, in order to extract the anticipated design proposal as a $2 \mathrm{D}$ map visualization. 


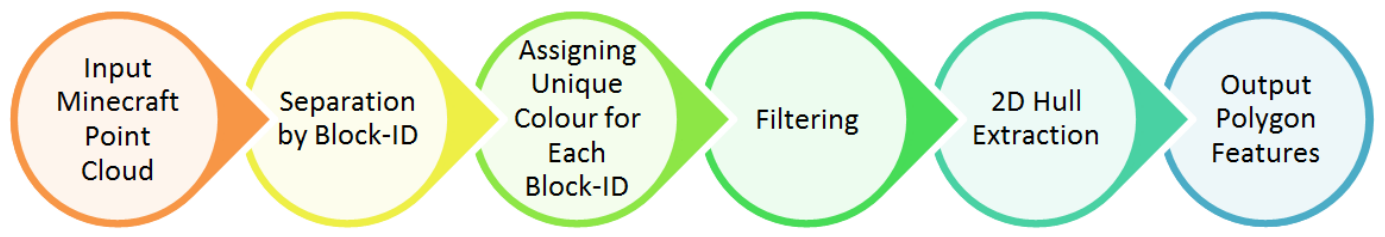

Figure 7: Post-processing workflow for retransforming Minecraft point cloud into 2D map visualization

The point cloud that is extracted from Minecraft contains block-ID and block-Data attributes for each point (Figure 8). Sorting points based on those attributes allows all the blocks and textures from Minecraft to be put into separate point clouds. Points with similar block-ID and block-Data attributes are combined in a single partial point cloud.
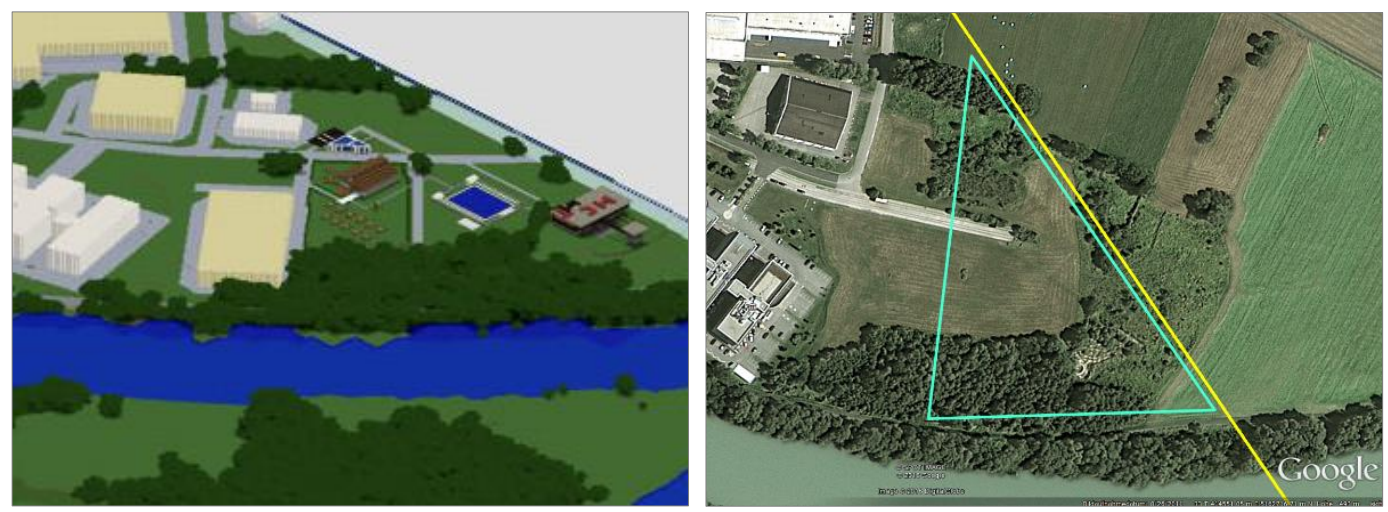

Figure 8: Virtual design proposal derived from public participation referenced by Google satellite image

For visualization and analysis purposes, each of these partial point clouds, with a certain block-ID, is given a unique colour. Next, the Minecraft point clouds are filtered in order to extract the vertices needed for classifying and mapping the design proposal. The last step in the post-processing workflow is extracting convex hull polygon features, which are used for visualization and the mapping of the design proposal (Figure 9). 


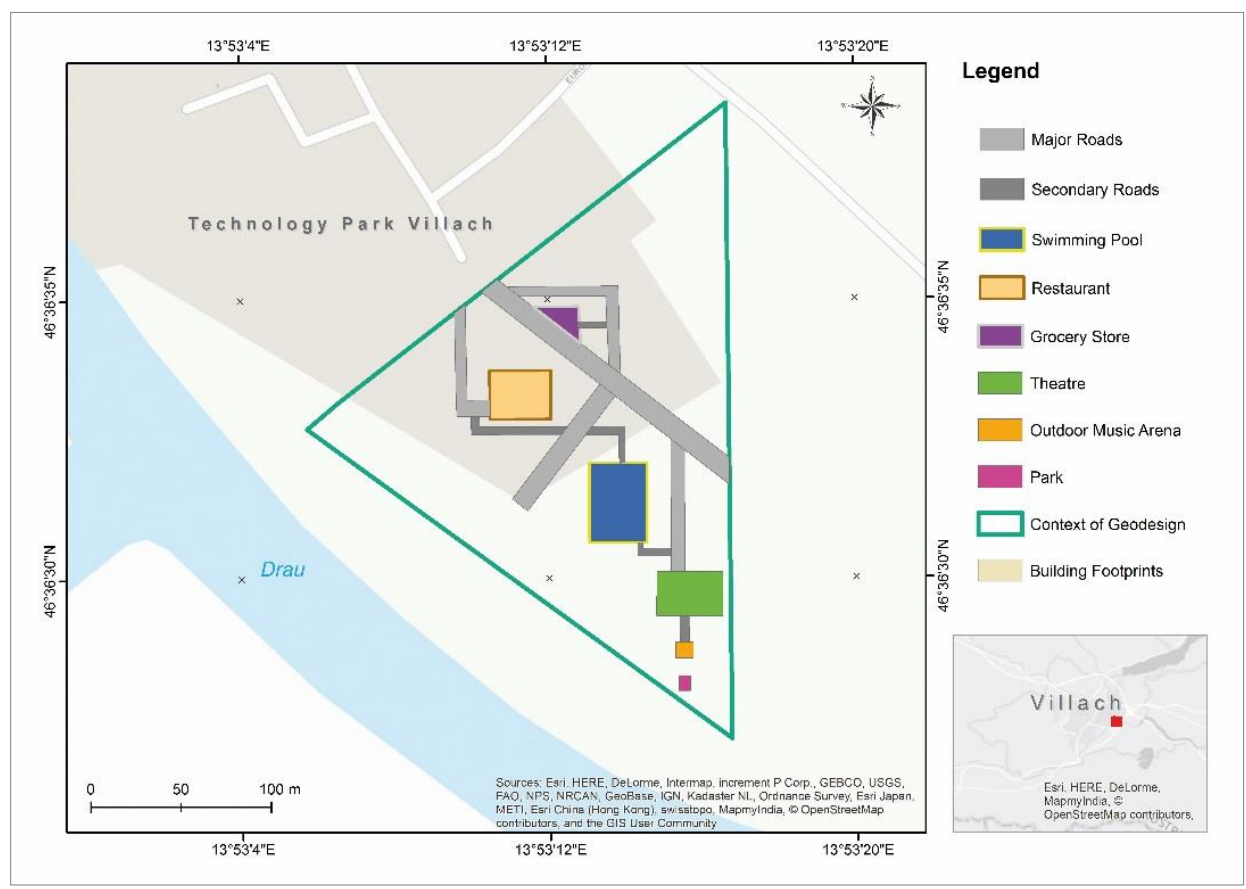

Figure 9: Mapping the design proposal derived from the Minecraft participatory geodesign process

\section{Future Work}

Steinitz (2014), with reference to geodesign workflow, states that a geodesign proposal that emerges from a collaborative design process should be evaluated using professional geodesign tools according to certain objective values of the urban planners and decision makers. The applicability of the design proposal should be assessed according to certain urban planning forces, depending on the society's needs and the availability of resources, in order to maintain sustainable urban planning (Campbell, 1996). The workflow introduced in this paper must be applied in multiple iterations in order to acquire several design scenarios that can reflect the needs of a wider range of the community in a specific region.

Further enhancement of the realism in Minecraft models can be achieved by integrating detailed 3D architecture models and 3D city GML models (Magnussen \& Elming, 2015), as well as Building Information Models (BIM) (Dmitribagh, 2015). Involving BIM in the data transformation methodology for the creation of Minecraft models could achieve further realism thanks to the possibility of direct translation of building materials, such as glass and walls, into equivalent Minecraft blocks. 


\section{$7 \quad$ Discussion and Conclusion}

This research project aimed to explore the usability of Minecraft for geodesign and for facilitating collaborative public participation in urban planning decision support. The main focus was the enhancement of the realism of the Minecraft prototype world by integrating multiple spatial datasets. The workflow for transforming actual high resolution geospatial data into a virtual Minecraft world introduced a gaming-based approach for urban planning, targeting young citizens and students to engage them in urban design.

Students from the university campus were invited to elaborate their design ideas for expanding the university campus. The targeted area as a context of geodesign is a public area, used only for parking, while it could be used for the expansion of the university campus. Evaluation of Minecraft's usability for geodesign and of the Minecraft prototype that was produced was based on the volunteer participants' feedback during the workshop through an online questionnaire.

The workshop participants showed remarkable interest in virtual building in Minecraft for first time. The training provided during the workshop allowed them to familiarize themselves with the game and to express their design ideas. However, building realistic models in Minecraft is time-consuming. The initiator of similar future events needs to take into account the participants' availability and to ensure a certain level of motivation (Brabham, 2009) to encourage them to contribute their individual input in urban design proposals.

The results showed that the Minecraft prototype produced in the project presented an adequate level of realism, due to the advanced integration and transformation of spatial datasets. This approach had a further positive influence in minimizing disorientation and improving the navigation experience in Minecraft. Using Minecraft as a geodesign tool was effective for generating individual urban designs. Utilizing the game capabilities efficiently offered techniques for improving communications and reducing conflicts among the participants in Minecraft. On the other hand, the drawback of Minecraft is that assessing the suitability of designs for urban planning is time-consuming and requires participants to have high levels of motivation.

This research project demonstrates that utilizing Minecraft as a gaming-based approach for urban planning purposes can result in the creation of reliable urban designs for possible implementation. Moreover, the research project shows how the automated transformation of spatial datasets to Minecraft might help engage the community to contribute in urban planning.

\section{Acknowledgements}

Laser scan data and high resolution orthophoto were provided by the Carinthian Geographical Information System KAGIS. Support from FME, Safe Software Inc., and constructive feedback on the prototype design by Dmitri Bagh- FME Scenario Creation and Testing Analyst are gratefully acknowledged. 


\section{References}

Amado, M. P., Santos, C. V., Moura, E. B., \& Silva, V. G. (2010). Public participation in sustainable urban planning. International Journal of Human and Social Sciences, 5(2), 102-108.

Balogh, G., \& Beszédes, A. (2013). CodeMetrpolis-A minecraft based collaboration tool for developers. In Software Visualization (VISSOFT), 2013 First IEEE Working Conference on (pp. 1-4). IEEE.

Brabham, D. C. (2009). Crowdsourcing the public participation process for planning projects. Planning Theory, 8(3), 242-262.

Breuer, J., Walravens, N., \& Ballon, P. (2014). Beyond defining the smart city. Meeting top-down and bottom-up approaches in the middle. Tema. Journal of Land Use, Mobility and Environment.

Campbell, S. (1996). Growing Cities, Just Cities? Urban Planning and the Contradictions of Sustainable Development. Journal of the American Planning Association, 62(3), 296-312.

Coudray, C. (2015). Minecraft in higher education, beyond learning activities. University of Edinburgh.

Denmark in Minecraft - Geo Minecraft (2016). Denmark in Minecraft - Geo Minecraft. Retrieved from http://www.geominecraft.com/denmark/

Dmitribagh, (2015). How to make Minecraft worlds, Safe Software Inc. Retrieved from https://knowledge.safe.com/articles/1064/how-to-make-minecraft-worlds.html

Duncan, S. C. (2011). Minecraft, beyond construction and survival. Well Played: a journal on video games, value and meaning, 1(1), 1-22.

Formosa, S. (2014). Neogeography and Preparedness for Real-to-Virtual World Knowledge Transfer: Conceptual Steps to Minecraft Malta. Future Internet, 6(3), 542-555.

Geofabrik GmbH (2015). OpenStreetMap Contributors, Map tiles, Creative Commons BY-SA 2. 0, and ODbL 1. 0, 'OpenStreetMap data for Austria'. In Geofabrik OpenStreetMap Data Extracts. Retrived from http://download.geofabrik.de/europe/austria.html

Helleborg, S., \& Åmdal, T. M. (2015). RealMaps: Using real maps in Minecraft.

Magnussen, R., \& Elming, A. L. (2015, October). Cities at Play: Children's Redesign of Deprived Neighbourhoods in Minecraft. In European Conference on Games Based Learning (p. 331). Academic Conferences International Limited.

Mayer, I. S., van Bueren, E. M., Bots, P. W., van der Voort, H., \& Seijdel, R. (2005). Collaborative decisionmaking for sustainable urban renewal projects: a simulation-gaming approach. Environment and Planning B: planning and design, 32(3), 403-423.

McElvaney, L. A., \& Foster, K. (2014). Enhancing Stakeholder Engagement: Understanding Organizational Change Principles for Geodesign Professionals. In Geodesign by Integrating Design and Geospatial Sciences (pp. 315-329). Springer International Publishing.

Minecraftinformation.com, (2012). Minecraft ID List. Retrieved from http://www.minecraftinformation.com/minecraftidlist

Rautenbach, V. J., Coetzee, S. M., \& Çöltekin, A. (2014). Towards evaluating the map literacy of planners in 2D maps and 3D models in South Africa. AfricaGEO 2014 Conference Proceedings.

Steinitz, C. (2014). Which Way of Designing?. In Geodesign by integrating design and geospatial sciences (pp. 11-40). Springer International Publishing.

von Heland, F., Westerberg, P., \& Nyberg, M. Using Minecraft as a citizen participation tool in urban design and decision making. 